\title{
A Numerical Approach for Modeling of Turbulent Newtonian Fluid Flow in Eccentric Annulus
}

\author{
Erman ÜLKER ${ }^{1}$ \\ Sıla Övgü KORKUT ${ }^{2}$ \\ Mehmet SORGUN ${ }^{3}$
}

\begin{abstract}
Turbulent flow is a complicated process that frequently appears not only in nature but also in engineering applications. Numerical methods frequently are used to solve turbulent flow problems due to the difficulty of solving Navier-Stokes equations. In this study, NavierStokes equations including inner pipe rotation effect are solved via two different techniques. A semi-analytical technique is described and applied to turbulent Newtonian fluid flow in an eccentric annulus with pipe rotation. The accuracy of the semi-analytical method is compared with the obtained numerical results of the Newton-Raphson method. The semi-analytical method is computationally time consuming, however, it may allow tackling the non-linearity of challenging problems in hydraulics. A mechanistic model including the semi-analytical method is developed in order to predict pressure gradient for turbulent flow through eccentric horizontal annulus with pipe rotation. The mechanistic model is discretisized using central difference approximation and then solved analytically in each iteration by freezing the nonlinear terms. Computational frameworks are developed in MATLAB. The mathematical model is confirmed by the experimental study, which is conducted in Izmir Katip Celebi University. Results show that computational fluid model is capable of estimating pressure gradient with an error of less than $14 \%$.
\end{abstract}

Keywords: Eccentric annular flow, finite difference method, mechanistic modeling, Newtonian fluid, pipe rotation, turbulent flow.

\section{INTRODUCTION}

Most of the water flow problems related to civil engineering are mostly turbulent. Turbulent flow is a complex and chaotic process encountered in many engineering disciplines. The main reason is complexity and difficulties of turbulent solutions of Navier- Stokes equations

Note:

- This paper has been received on September 05, 2017 and accepted for publication by the Editorial Board on April 17, 2018.

- Discussions on this paper will be accepted by September 30, 2018.

- DOI: $10.18400 /$ tekderg.336801

1 Izmir Katip Celebi University, Dep. of Civil Engineering, İzmir, Turkey - erman.ulker@ikc.edu.tr

2 Izmir Katip Celebi University, Engineering Sciences, İzmir, Turkey - silaovgu.korkut@ikc.edu.tr

3 Izmir Katip Celebi University, Dep. of Civil Engineering, İzmir, Turkey - mehmetsorgun@gmail.com 
which hinder analytical demonstration. Even a simple problem in turbulent fluid flow is of concern regarding existence and uniqueness of the solution.

In applications of fluid flow through the annulus, the geometry is rarely concentric. Most of them lose the alignment of the center body and become eccentric due to gravity, bending etc. Fluid flow in an eccentric annulus has attracted great attention of researchers over the years. Deissler [1] investigated an analytical model for turbulent heat and mass transfer in smooth tubes. He took into account the effect of kinematic viscosity in the region close to the wall and obtained good agreement with experimental results. After his previous work, Deissler and Taylor [2] conducted an analytical study to describe the velocity profile of turbulent flow through eccentric annular geometries. Wolffe and Clump [3] conducted an experimental study determining velocity lines and locus of maximum velocity for turbulent flow of air. They compared their experimental results with the calculated solution of Navier-Stokes equation with Heyda's assumption [4] for analytical solution of laminar flow in annuli containing eccentricity. Johnson and Sparrow [5] [6] conducted experimental work on turbulent flow in eccentric annulus and they reported that circumferential pressure gradient is larger than that of concentric annulus. Also, they observed that friction factor decreases with increasing eccentricity. Rehme [7] proposed a correlation of friction factor prediction for turbulent flow in channels with non-circular cross-sections. He concluded that this prediction method is better than all previous attempts and it can be used on other shapes of channels such as eccentric annulus. Kacker [8] conducted an experimental study of fully turbulent flow in a circular pipe containing one or two eccentrically located rods. He developed a correlation to estimate friction factor. The correlation predicts the experimental data with $2 \%$ error margin for both one and two rod geometries. Usui and Tsuruta [9] analyzed the equation of motion for fully turbulent flow in an eccentric annulus using Kirchoff transformation. They explained the dependence of eccentricity on friction factor at high Reynolds number ranges. Tosun [10] proposed an approximate solution axial laminar flow through the eccentric annulus and he compared his results with previously published experimental works. The approximate solution is relatively in good agreement with the data in the literature. He and his group [11] [12] expanded the study of non-Newtonian fluids applying the Power law, Bingham plastic and Sutterby models. Those studies concluded that proposed approximate solution is in good agreement with previous experimental studies. Ogino et al. [13] also investigated the momentum equation for fully developed turbulent flow in the eccentric annulus. They used the bipolar coordinate transform in order to model the eccentric annulus geometry. Haciislamoglu and Langlinais [14] investigated the effect of eccentricity on frictional pressure losses without inner pipe rotation and obtained pressure losses reduction as high as $60 \%$. Nouri et al. [15] conducted an experimental study on Newtonian and non-Newtonian fluids in both concentric and eccentric annulus. They concluded that the flow resistance decreases as the eccentricity is increased as compared with smooth pipe flow. One year later, Nouri and Whitelaw [16] introduced rotational effect in the same study and their results showed that the effect of rotation on frictional pressure losses decreases with increase in Reynolds number after the flow is in transition. Decreasing of rotational effect on frictional pressure losses continues until they are equal to the non rotation case. Numerical and analytical approaches have been performed for modelling turbulent flow. The study of Haciislamoglu and Langlinais [14] was conducted under the laminar flow regime. Corresponding to this study, Erge et al. [17] conducted another experimental study of turbulent flow in the eccentric annulus. They concluded that eccentricity significantly 
reduces the frictional pressure loss in turbulent flow as well. Mossa [18] conducted an experimental study of turbulent flow, which averaged Reynolds number in the range of $[10,000 ; 30,000]$. He applied statistical analysis to find velocity fluctuations around the local averaged value. Erge et al. [19] conducted an experimental, analytical and numerical study of the effect of eccentricity on frictional pressure loss. They concluded that frictional pressure loss in fully eccentric annulus has up to $50 \%$ discrepancy than the results which is calculated with Narrow Slot approach. Recently, Rushd et al [20] investigated eccentricity, roughness and rotation effects on frictional pressure loss. They conducted an experimental study and developed a CFD model in ANSYS validated by experimental results. Their CFD analysis showed that effect of roughness and eccentricity are more prominent than the effect of inner pipe rotation.

This study aims to develop a mechanistic model including a semi-analytical method for determining accurate pressure gradient of turbulent flow through the fully eccentric annulus, including the effect of pipe rotation. The semi-analytical method presents the discretization of the equation with finite difference method and solved iteratively by fixing the non-linear terms.

\section{EXPERIMENTAL METHOD}

A flow loop was constructed at Izmir Katip Celebi University, Civil Engineering, Fluid Mechanics \& Hydraulics Laboratory. A Newtonian fluid, water, has been used in the experiment. Flow loop, $10 \mathrm{~m}$ long, has been formed using $80 \mathrm{~mm}$ diameter outer pipe and 40 $\mathrm{mm}$ diameter inner pipe. The inner pipe is chromium plated and the outer pipe is made of plexiglass. In this study, both pipes are assumed smooth. In the present experimental study, Newtonian fluid flows through the annulus that is between inner and outer pipes. The inner pipe has been fixed concentrically at both ends and full eccentric annulus has been obtained at the test section. The test section is $0.5 \mathrm{~m}$ long and $7 \mathrm{~m}$ away from the upstream due to obtaining a fully developed section. A picture of the flow loop is shown in Fig. 1 and the specifications of the experimental setup are given in Table 1.

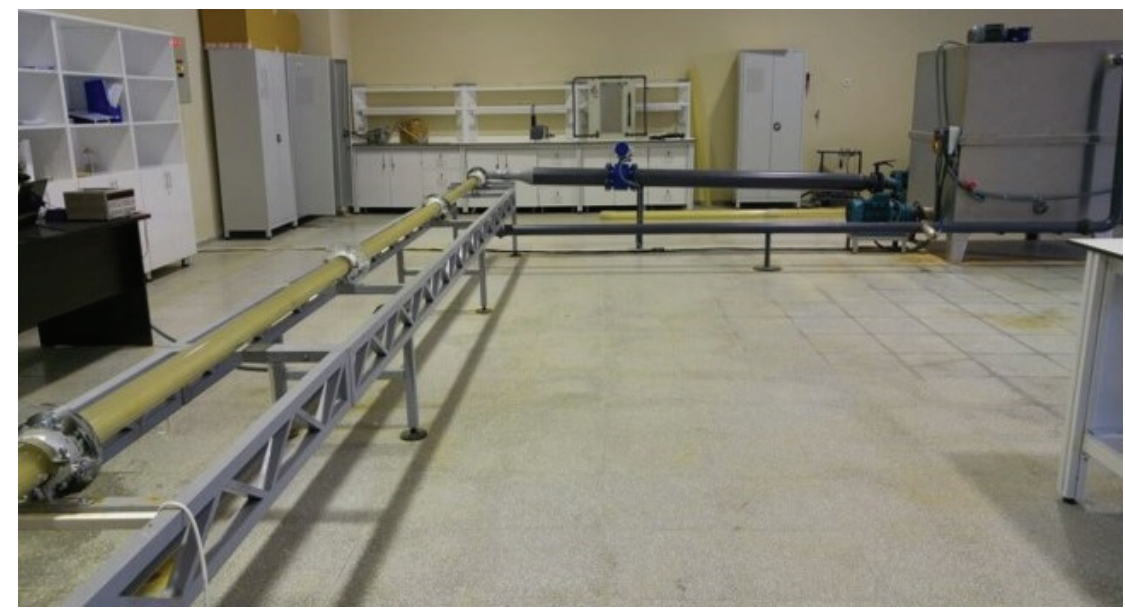

Figure 1. Izmir Katip Celebi University Civil Engineering (IKCU-CE) Flow Loop 
Table 1. Test Parameter Values During Experiments

\begin{tabular}{cc}
\hline Experiment Specifications & Values \\
\hline Inner - Outer Pipe Diameter & $40-80 \mathrm{~mm}$ \\
Flow Loop length & $10 \mathrm{~m}$ \\
Flow velocity & $0.7-3.6 \mathrm{~m} / \mathrm{s}$ \\
Inner pipe rotation speed & $0-120 \mathrm{rpm}$ \\
\hline
\end{tabular}

The following procedure is used for the tests:

- Fill the feeding tank with water

- Start the thermostatic heater for increasing the temperature of the fluid in the feeding tank

- Set the heater to desired value by digital controller

- Start $230 \mathrm{~V}$ DC motor on the tank for stirring the fluid in the tank in order to have homogenous temperature distribution in the fluid

- Open the butterfly valve to release the fluid to the system

- Start 10 HP pump

- Wait till the flow is steady and isothermal in the entire system

- Set flow rate to desired value

- Start 230 V DC motor for rotating inner pipe

- Set inner pipe rotation rate to desired value

- Start data acquisition system

- Start 0-50V / 0-20 A switching power supply

- Connect ETRANS-M 210 electromagnetic flowmeter to the power supply

- Connect ETRANS-DP pressure transmitter to the power supply

- Start recording data

- As soon as the readings are stable, change one of the input parameters (flow rate, inner pipe rotation rate, temperature)

- Repeat the previous step until data is collected for all desired parameters.

- Stop recording data

- Disconnect flowmeter and pressure transmitter

- Stop power supply

- Stop the motor for inner pipe rotation

- Stop the pump

- Stop the motor for stirring the fluid inside the feeding tank

- Stop the heater

The detail of experimental tools and complete data set can be seen by interested readers in the previous work [21]. When the system is completely filled with the fluid, a motor pump of $10 \mathrm{HP}$ is switched on. To make rotation pipes ready for rotating, $230 \mathrm{~V}$ DC motor is turned 
on as well. Furthermore, a 0-50V / 0-20 A switching power supply is used in order to switch on ETRANS-M 210 electromagnetic flowmeter and ETRANS-DP pressure transmitter which are connected to a desktop PC via a DT80 data logger. Data logger retrieves the data each 5 seconds from the system and the data is stored in 5 minutes intervals for each flow condition. The pressure gradient and flow rate are recorded by taking their average during 5 minutes. The fluid is arranged to attain the desired flow value in the system by using the butterfly valve. Reynolds number of the system is between $3.8 \times 10^{4}-1.08 \times 10^{5}$. Then, the digital controller of the inner pipe rotation motor is set to the desired value of rotational speed as well. The fluid is allowed to run through the system for a certain time till the flow becomes steady and isothermal in the entire system. As soon as the readings are stable, experimental data recording is started. Input parameters are changed accordingly and repeated as in previous steps until data is collected for all desired parameters.

\section{MECHANISTIC MODEL}

Navier-Stokes equations for turbulent flow including pipe rotation in Cartesian Coordinate in an open form is formulated without assumptions as; (Bird et al. [22])

For the x-direction;

$$
\begin{aligned}
& \rho\left(\frac{\partial u}{\partial t}+u \frac{\partial u}{\partial x}+v \frac{\partial u}{\partial y}+w \frac{\partial u}{\partial z}\right)= \\
& -\frac{\partial P}{\partial x}+\left[\frac{\partial}{\partial x} \tau_{x x}+\frac{\partial}{\partial y} \tau_{y x}+\frac{\partial}{\partial z} \tau_{z x}\right]+\rho g_{x}-\frac{\partial\left(\rho u^{\prime 2}\right)}{\partial x}-\frac{\partial\left(\rho u^{\prime} v^{\prime}\right)}{\partial y}-\frac{\partial\left(\rho u^{\prime} w^{\prime}\right)}{\partial z}+\rho \omega^{2} y .
\end{aligned}
$$

For the y-direction;

$$
\begin{aligned}
& \rho\left(\frac{\partial v}{\partial t}+u \frac{\partial v}{\partial x}+v \frac{\partial v}{\partial y}+w \frac{\partial v}{\partial z}\right)= \\
& -\frac{\partial P}{\partial y}+\left[\frac{\partial}{\partial x} \tau_{x y}+\frac{\partial}{\partial y} \tau_{y y}+\frac{\partial}{\partial z} \tau_{z y}\right]+\rho g_{y}-\frac{\partial\left(\rho u^{\prime} v^{\prime}\right)}{\partial x}-\frac{\partial\left(\rho v^{\prime 2}\right)}{\partial y}-\frac{\partial\left(\rho v^{\prime} w^{\prime}\right)}{\partial z} .
\end{aligned}
$$

For the z-direction;

$$
\begin{aligned}
& \rho\left(\frac{\partial w}{\partial t}+u \frac{\partial w}{\partial x}+v \frac{\partial w}{\partial y}+w \frac{\partial w}{\partial z}\right)= \\
& -\frac{\partial P}{\partial z}+\left[\frac{\partial}{\partial x} \tau_{x z}+\frac{\partial}{\partial y} \tau_{y z}+\frac{\partial}{\partial z} \tau_{z z}\right]+\rho g_{z}-\frac{\partial\left(\rho u^{\prime} w^{\prime}\right)}{\partial x}-\frac{\partial\left(\rho v^{\prime} w^{\prime}\right)}{\partial y}-\frac{\partial\left(\rho w^{\prime 2}\right)}{\partial z} .
\end{aligned}
$$

Necessary assumptions are assigned to simplify Navier-Stokes equations. The following assumptions are taken into account;

- fully developed turbulent flow

- steady state 
- $\quad$ incompressible fluid

Moreover, Erge et al. [23] take into account other assumptions, which are no gravitational effects and an isothermal system. So, Navier-Stokes equations for Newtonian fluids in a narrow slot can be written as;

$\frac{\partial}{\partial y}\left[\left(\mu+\mu_{t}\right) \frac{\partial u}{\partial y}\right]=\frac{\partial P}{\partial x}+\rho \omega^{2} y$.

In order to define turbulent viscosity, Prandtl's Mixing Length Hypothesis has been used in the literature [24]. Turbulent viscosity can be formulated as;

$\mu_{t}=\rho l_{m}^{2}\left|\frac{\partial u}{\partial y}\right|$

The mixing length can be expressed in the van Driest formula;

$l_{m}=\kappa y$

where $\kappa$ is von Karman constant and its value is 0.4 . The viscous effect is significant close to the wall and mixing length approaches zero at the wall. In order to account for this behavior, the viscous damping function could be added to the van Driest's formula;

$l_{m}=\kappa y\left(1-e^{-\frac{y^{+}}{A}}\right)$

where $y^{+}=\frac{y \sqrt{\tau_{w} \rho}}{\mu}$ and $\tau_{w}=-H \frac{\partial P}{\partial x}$. Here $A$ represents van Driest's damping constant and it has been assumed to be 26 for smooth surfaces without suction or blowing. $H$ is the half height of the annulus.

It is now obvious that both $l_{m}$ and $\mu_{t}$ are the function of $y$. Chain rule method will be applied step by step to the final version of momentum equation for slot flow approximation. It can be seen in Eq. (8). Then, to solve the obtained equation, the finite difference algorithm is used.

$\left(\mu+\mu_{t}\right) \frac{\partial^{2} u}{\partial y^{2}}+2 \rho l_{m} \frac{\partial l_{m}}{\partial y}\left(\frac{\partial u}{\partial y}\right)^{2}=\frac{\partial P}{\partial x}+\rho \omega^{2} y$

On both inner and outer pipe surfaces $(y=0$ or $y=2 H)$, it is assumed to exist a non-slip boundary condition. Due to symmetrical geometry of fluid flow, the boundary condition is taken only at the bottom of the channel as $u(y=0)=0$. On the other hand, there is no shear stress at the center of the flow in the annulus. In other words, the maximum velocity of the flow is reached at the center of the flow profile. In mathematical representation of this boundary condition is $\frac{d u}{d y}(y=H)=0$.

In the present study, experiments were conducted for flow through the fully eccentric annulus. Therefore, the height of the annulus should be determined in view of the eccentric 
annulus. Previous researchers used complex coordinates transformation, conformal mapping or computational iterations. Iyoho and Azar [25] proposed a model for determining height of eccentric annulus with a simple formula;

$2 H=\sqrt{r_{o}^{2}-\varepsilon^{2} c^{2} \sin ^{2} \theta}-r_{i}+\varepsilon \cos \theta$

\subsection{Numerical Methods}

In order to discretisize derivatives of the Eq. (8), the central difference scheme is used;

$$
\begin{aligned}
& \frac{\partial u}{\partial y}=\frac{u_{i+1}-u_{i-1}}{2 \Delta y} \\
& \frac{\partial^{2} u}{\partial y^{2}}=\frac{u_{i+1}-2 u_{i}+u_{i-1}}{(\Delta y)^{2}}
\end{aligned}
$$

To proceed with the computer programming, derivative operators are needed to be put into matrix form by using the above formulas with accounting boundary conditions. Then, it leads to

$$
\begin{gathered}
\frac{\partial}{\partial y}=D_{1}=\frac{1}{2 \Delta y}\left(\begin{array}{cccccc}
0 & 1 & 0 & \cdots & \cdots & 0 \\
-1 & 0 & 1 & 0 & \cdots & 0 \\
0 & -1 & 0 & \ddots & \ddots & \vdots \\
\vdots & 0 & \ddots & \ddots & 1 & 0 \\
\vdots & \vdots & \ddots & -1 & 0 & 1 \\
0 & 0 & \cdots & 0 & -1 & 0
\end{array}\right) \\
\frac{\partial^{2}}{\partial y^{2}}=D_{2}=\frac{1}{(\Delta y)^{2}}\left(\begin{array}{cccccc}
-2 & 1 & 0 & \cdots & \cdots & 0 \\
1 & -2 & 1 & 0 & \cdots & 0 \\
0 & 1 & -2 & \ddots & \ddots & \vdots \\
\vdots & 0 & \ddots & \ddots & 1 & 0 \\
\vdots & \vdots & \ddots & 1 & -2 & 1 \\
0 & 0 & \cdots & 0 & 1 & -2
\end{array}\right)
\end{gathered}
$$

where $\mathrm{D}_{1}$ and $\mathrm{D}_{2}$ are $(\mathrm{N}-1) \times(\mathrm{N}-1)$ matrices in which $\mathrm{N}$ represents the number of discretization in the numerical solution. Therefore, the final version of the discretisized momentum equation for slot flow is as follows;

$$
\left(\vec{\mu}+2 \vec{\mu}_{t}\right) D_{2} \vec{u}+2 \rho \vec{l}_{m} D_{1} \vec{l}_{m}\left(D_{2} \vec{u}\right)^{2}=\vec{P}_{x}
$$

Due to the symmetricity of the computational domain, Eq. (14) has been solved by using two approaches on the interval $[0, H]$ by dividing $N$ equal sub-intervals. 


\subsection{A Semi-Analytical Method}

The main idea of the semi-analytical method is based on frozen non-linear term. After getting rid of the non-linearity and embedding the matrices defined in Eq. (12) and (13) into Eq. (8), it is transformed into an algebraic equation such that

$A \vec{U}=B$

which is solved analytically. In literature Vidts and White [26] proposed a semi-analytical method for solving a linear partial differential equation. The authors also discretisized the space by considering central difference approximation and then an ordinary differential

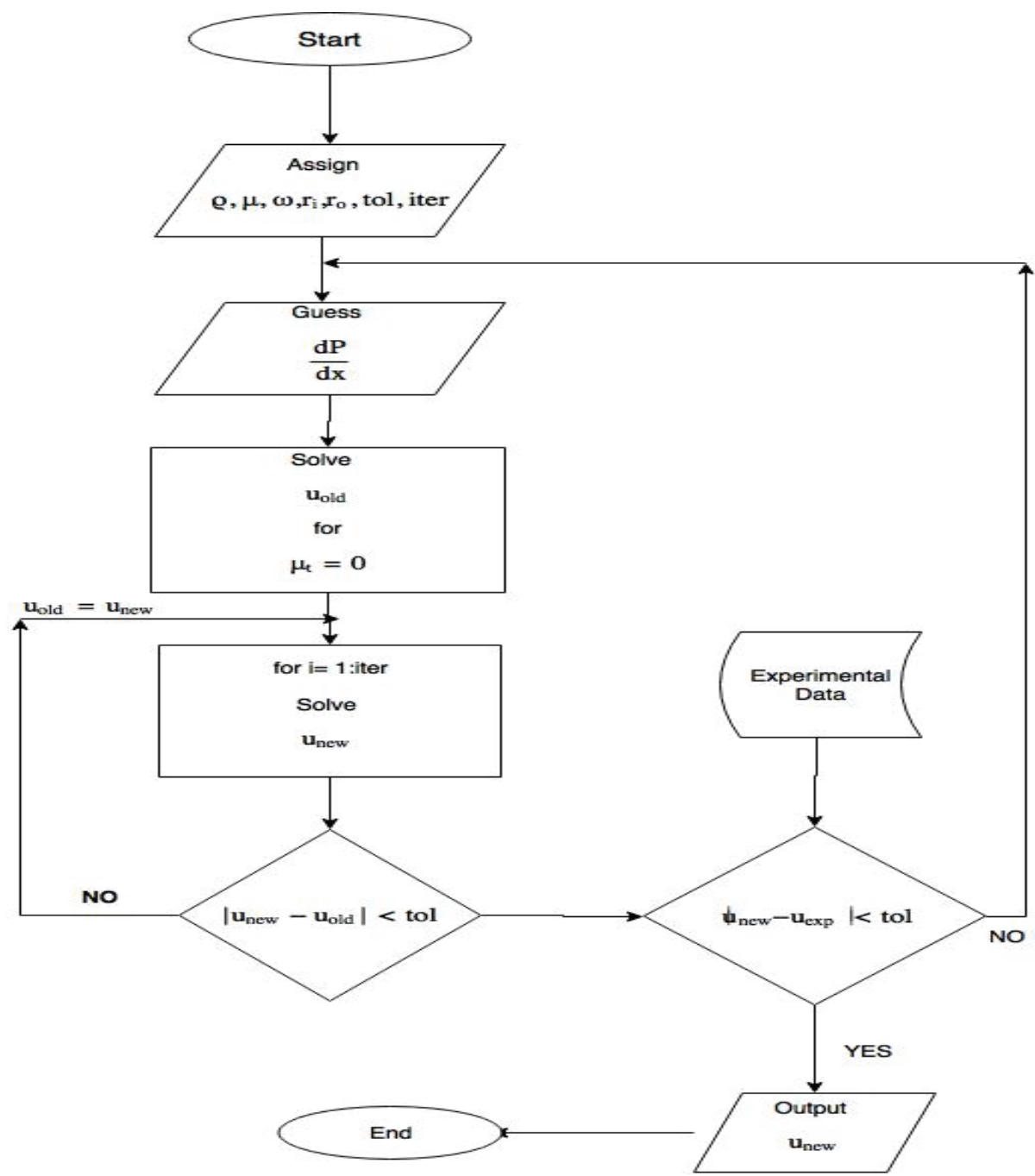

Figure 2. Flowchart of the computer code with new approach 
equation is obtained which is solved analytically. Moreover, detailed discussion on the applications of semi-analytical methods for fluid flows can be found in [27]. One of the famous techniques for the semi-analytical solution is Adomian decomposition method. However, to obtain a series expansion the technique has tedious calculations therein. In the present study, an alternative semi-analytical method is suggested.

The process of the semi-analytical method is organized as follows: The initial assumption of the velocity profile is obtained by considering it laminar where the turbulent viscosity is assigned the value of zero. This assumption is taken as an old value of the velocity. In the iterative process, obtaining the new solution the non-linear term is frozen by using the old solution. After freezing the non-linear term the solution is obtained analytically. In order to obtain the final solution, the accuracy of the solution is checked by a given tolerance. If the tolerance is not attained, then it is returned back to the iterative loop by assigning the new solution as an old solution. Otherwise, the new solution is assigned as the pre-final solution. Then, the pre-final solution is compared with experimental data. If the error between the prefinal solution and experimental data is less than the given tolerance then the pre-final solution is assigned as the final solution. Otherwise, the program returns to assign initial assumptions of the pressure gradient. The flow chart of this program can be seen in Fig. 2.

\subsection{Newton-Raphson Method}

After getting an algebraic non-linear equation the question leads to finding the root instead of the solution. Thus, Newton-Raphson method is the well-known and accurate non-linear solver among engineers and scientists for finding the root of $F(\vec{u})=0$. Thus, NewtonRapson method is used to check the validity of the solutions obtained via the semi-analytical method. The process of Newton's algorithms is defined as

$\vec{u}_{n+1}=u_{n}-\left(F^{\prime}\left(\vec{u}_{n}\right)\right)^{-1} F\left(\vec{u}_{n}\right)$

where $F: \mathbb{R}^{n} \rightarrow \mathbb{R}^{n}$ and $F(\vec{u}) \in C^{2}\left(\mathbb{R}^{n}\right)$. By fixing $\vec{u}_{n}$ the above equation becomes a linear equation even though $F($.$) is non-linear. It is worth mentioning that the choice of the initial$ assumption, $\vec{u}_{0} \in \mathbb{R}^{n}$, is a key point for both avoiding the singularity problem of $\left(F^{\prime}\left(\vec{u}_{n}\right)\right)^{-1}$ and also the convergence of the method. After doing tedious calculations we obtain

$F\left(\vec{u}_{n}\right)=\left(\mu+2 \mu_{t}\right) D_{2} \vec{u}_{n}+2 \rho l_{m} D_{1} l_{m}\left(D_{1} \vec{u}\right)^{2}-\vec{P}_{x}$.

Here, $F^{\prime}(\vec{u})$ denotes the derivative of $F(\vec{u})$ with respect to $\vec{u}$. Thus, the derivative of $F(\vec{u})$ in operator form is as follows:

$F^{\prime}(\vec{u})=\left(\mu+2 \mu_{t}\right) D_{2}+2 \rho l_{m}\left(D_{1} l_{m}\left(2 D_{1} \vec{u}\right)+l_{m} D_{2} \vec{u}\right)$.

The operator forms defined in Eq. (16) and Eq. (17) are substituted into Newton-Raphson method given in Eq. (15). This equation is solved for $\vec{u}_{n+1}$. In the computational process of this approach, the initialization of the velocity, $\vec{u}_{0}$, is assigned as zero vector and this initialization vector is called the old solution. In the iterative process, solve Eq. (15) for getting the new solution for the velocity. Then, check the error of the solutions between the 
new and the old solutions by a given tolerance. If the tolerance is not attained, then return to the iterative loop by assigning the new solution as an old solution. Otherwise, the new solution is assigned as the pre-final solution. Then, the pre-final solution is compared with experimental data. If the error between the pre-final solution and experimental data is less than the given tolerance then the pre-final solution is assigned as the final one. Otherwise, the program returns to assignation of the initial assumption of the pressure gradient.

\subsection{Comparison of Both Methods}

The computer codes are tested for $60 \mathrm{rpm}$ inner pipe rotation and random pressure gradient. In Table 2, it can be seen that the discrepancy between Newton's method and the semianalytical method is insignificant. Therefore, other important parameter-computational costis checked for the same procedure. Table 3 shows time elapsed values for each method for

Table 2. Comparison of Two Numerical Approches

\begin{tabular}{cccc}
\hline & $\begin{array}{c}\text { Pressure Gradient } \\
(\mathrm{Pa} / \mathrm{m})\end{array}$ & $\begin{array}{c}\text { Pressure Gradient } \\
(\mathrm{Pa} / \mathrm{m})\end{array}$ & $\begin{array}{c}\text { Pressure Gradient } \\
(\mathrm{Pa} / \mathrm{m})\end{array}$ \\
\cline { 2 - 4 } Velocity $(\mathrm{m} / \mathrm{s})$ & Newton's Method & $\begin{array}{c}\text { Semi-Analytical } \\
\text { Method }\end{array}$ & Experimental Result \\
\hline 1.09 & 354.112 & 354.108 & 312.074 \\
\hline 1.41 & 526.893 & 526.881 & 510.901 \\
\hline 1.74 & 732.615 & 732.622 & 731.447 \\
\hline 2.44 & 1254.433 & 1254.456 & 1258.121 \\
\hline 2.71 & 1539.298 & 1539.32 & 1578.124 \\
\hline 3.37 & 2236.169 & 2236.179 & 2239.762 \\
\hline
\end{tabular}

Table 3. Computational Cost For Two Models Used In The Present Study For Calculating Frictional Pressure Losses At Same Input Parameters

\begin{tabular}{cc}
\hline \multicolumn{2}{c}{ Elapsed Time (second) } \\
\hline Newton's Method & $\begin{array}{c}\text { Semi-Analytical } \\
\text { Method }\end{array}$ \\
\hline 8.8 & 20 \\
8.6 & 28 \\
8.7 & 34 \\
9.3 & 39 \\
9.4 & 43 \\
9.5 & 56 \\
10.6 & 69 \\
\hline
\end{tabular}


predicting average velocity at the same input parameters. It is seen that the semi-analytical method takes relatively longer time than the Newton's method. The mathematical content of hydraulics applications is far from trivial. Comprehensive understanding of the physical behavior of fluid flow is required for getting accurate solutions. Just recently, methods have been eventually available to predict all features of annular flow including its affecting parameters such as flow velocity, rotating cylinder, eccentricity while numerical approaches have been developing with technological enhancement.

\section{RESULTS AND DISCUSSION}

In the present study, a semi-analytical method is applied to fully developed turbulent Newtonian fluid flow in the eccentric annulus with pipe rotation. Because of the nonavailability of the exact solution, the solutions obtained via the semi-analytical method is compared with a well-known numerical method, Newton-Raphson method, in terms of applicability and accuracy. However, the semi-analytical method takes relatively longer time than Newton's method. It is observed that increasing step size over 1000 makes no difference in pressure gradient for all cases. In other words, the step size is formulated as $\Delta y=\frac{H}{1000}$. Although the semi-analytical method is computationally expensive, it may help researchers to overcome the non-linearity of challenging problems in order to have the semi-analytical solution. The momentum equation of fully developed turbulent flow including inner pipe rotation in curvilinear coordinates, such as Bipolar coordinate that is one of the adequate coordinate systems for eccentric annulus geometry, is a good example of this challenging problem.

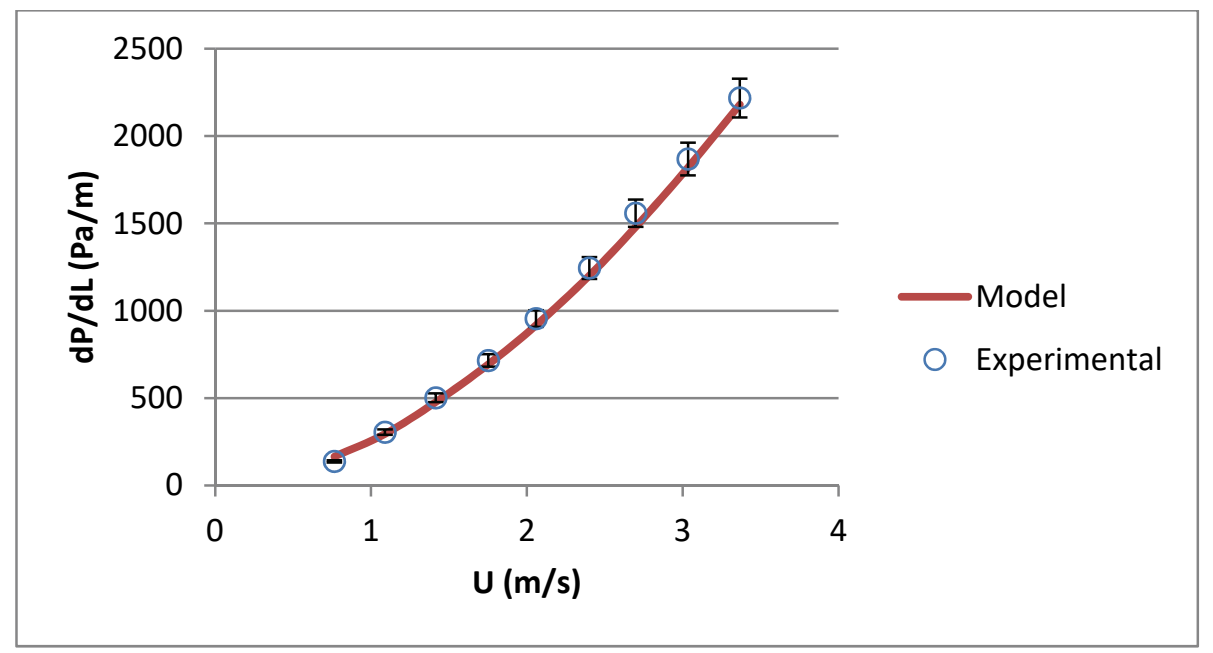

Figure 3. Comparison of measured and predicted pressure gradient without inner pipe rotation 
After setting up all the assumptions and constant values, the results are obtained within a reasonable accuracy when it is compared with experimental data. Uncertainty analysis is used for determining the experimental uncertainties corresponding to experimental data. General uncertainty analysis using the Taylor series method is used. The previous study of Ulker et al. [21] is presented that the maximum uncertainty error for determining pressure gradient is around 5\%. That means that the correlation has developed with a 95\% level of confidence. Fig. 3 shows predicted and measured data for pressure gradient in fully eccentric annulus at room temperature without inner pipe rotation.

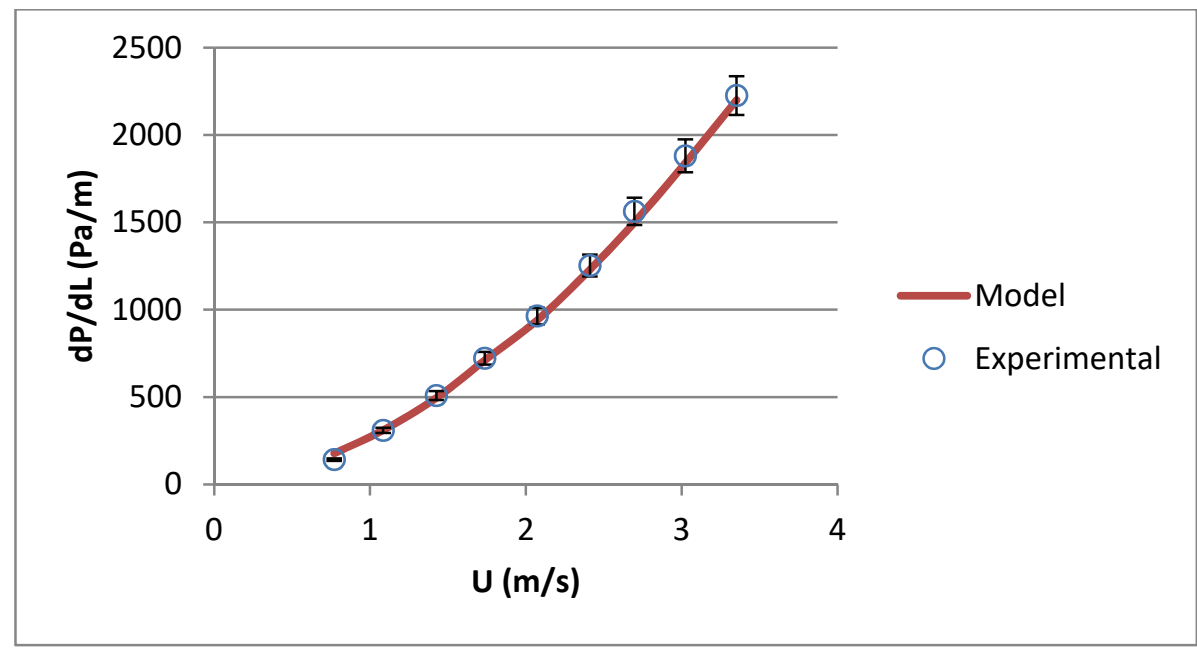

Figure 4. Comparison of measured and predicted pressure gradient for 30 rpm inner pipe rotation

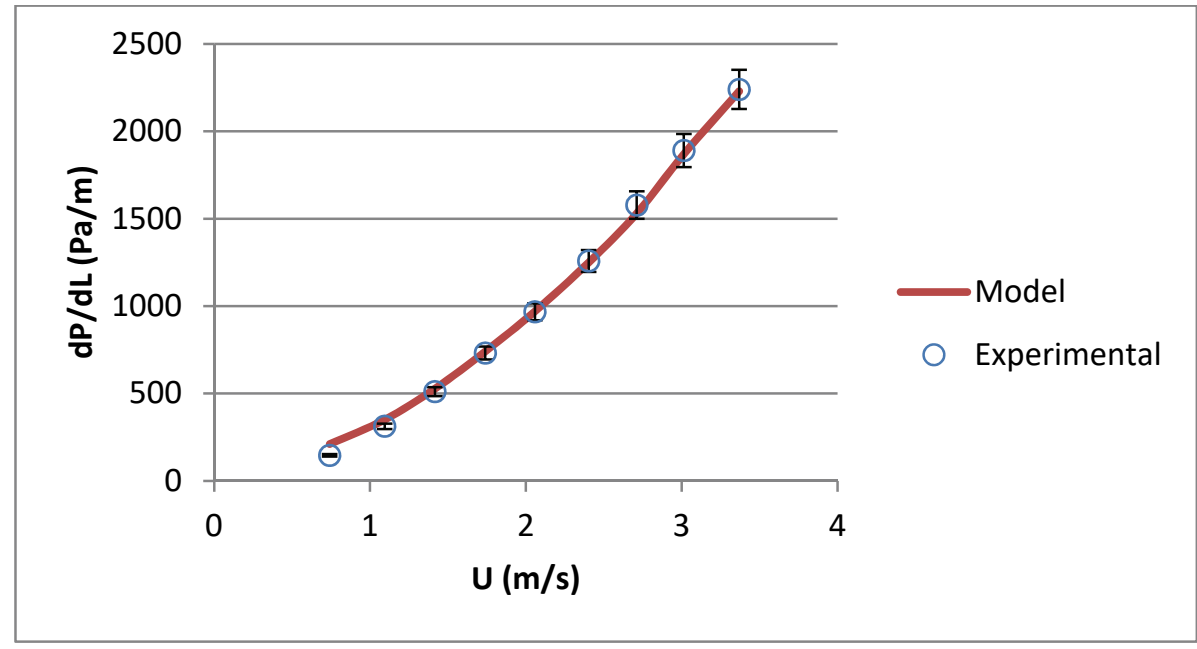

Figure 5. Comparison of measured and predicted pressure gradient for 60 rpm inner pipe rotation 


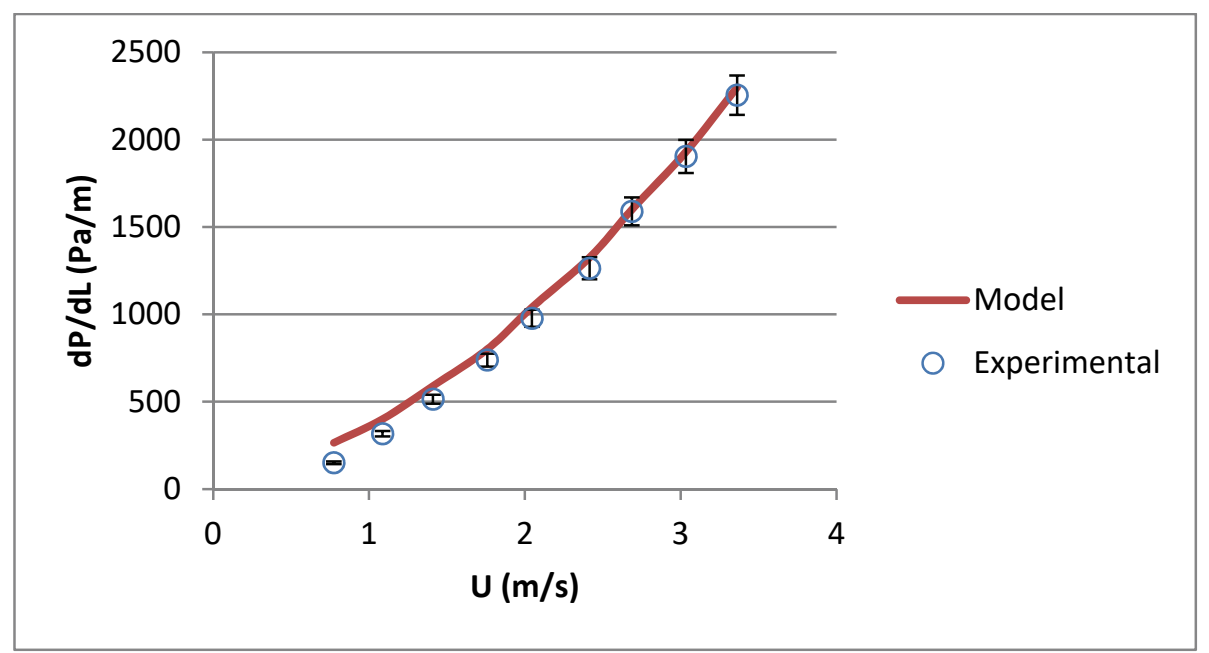

Figure 6. Comparison of measured and predicted pressure gradient for 90 rpm inner pipe rotation

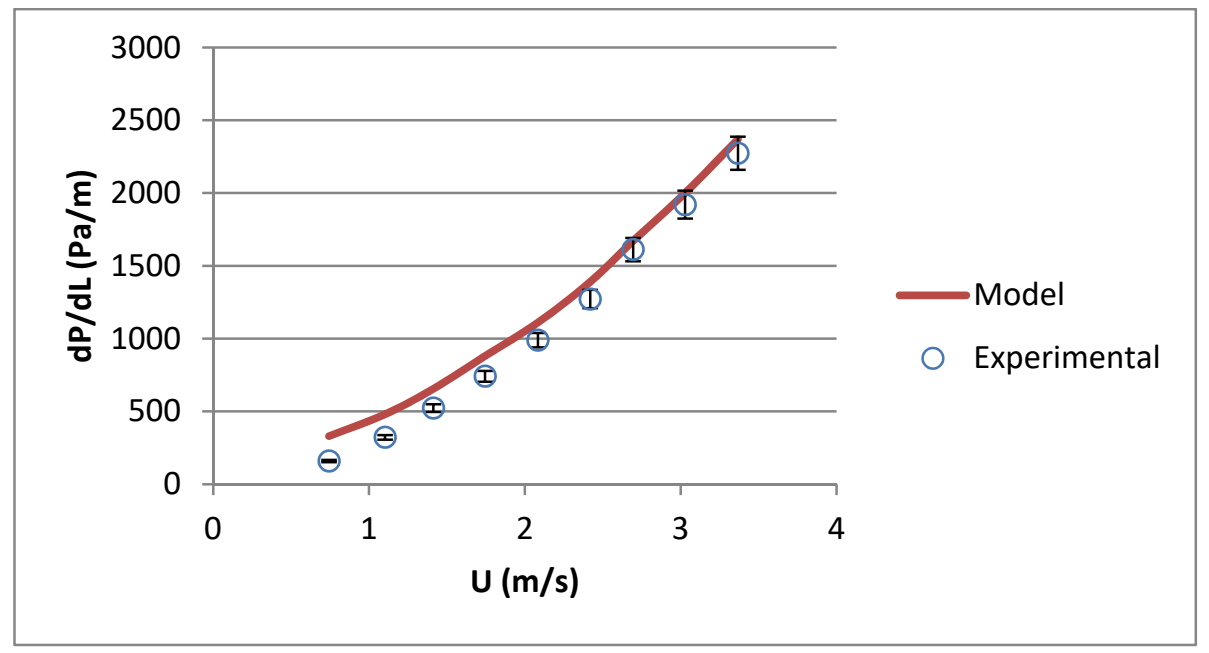

Figure 7. Comparison of measured and predicted pressure gradient for $120 \mathrm{rpm}$ inner pipe rotation

The proposed model predicted the pressure gradient within $5.3 \%$ error margin. This shows the model has good accuracy. From now on, rotation effects can be included and compared. Figs. 4-7 show the pressure gradient for different inner pipe rotation rates.

When the Figs. 4-7 are analyzed, it can be concluded that the model can predict better pressure gradient at higher velocities. At lower velocities and high inner pipe rotation rate, 
the model prediction performance is deteriorating. The reason is the wobbling effect of the inner pipe due to concentric ends. The axial velocity couldn't overcome this effect at lower velocities. Therefore, the discrepancy between the results arises.

The proposed mechanistic model gives reasonably close values with respect to experimental measurements. To have better insight, the performance of the methods is measured by calculating Average Absolute Percent Error (AAPE) and the result shows that pressure gradient for Newtonian fluid flow in fully eccentric annulus without inner pipe rotation and at room temperature can be predicted within a $5.3 \%$ error margin by using the semi-analytical method. However, the error increases as the effect of pipe rotation take part in the flow. Nevertheless, the model can determine a pressure gradient up to $14 \%$ error margin for all cases.

\section{CONCLUSION}

In the present study, two different methods are used to solve Navier-Stokes equations including inner pipe rotation effects and turbulent flow. The equation is primarily discretisized by the semi-analytical method via the central difference approximation and solved analytically in each iteration by freezing the non-linear terms. Even though the nonlinear terms are frozen in each iteration, the fixed terms are renewed by the previous solution in each step. In other words, pressure gradient and the turbulent viscosity are solved sequentially. The results of the semi-analytical method are confirmed by a well-known "Newton-Raphson method". Although the former is computationally expensive, this numerical technique may allow solving challenging problems in a semi-analytical way. Additionally, the semi-analytical method predicts pressure gradient successfully. A mechanistic model is developed using the semi-analytical method for fully developed turbulent flow through eccentric annulus including inner pipe rotation. When the developed mechanistic model is compared with experimental measurements, it gives reasonably good agreement. Results show that without rotation, the developed model can determine pressure gradient within a 5.3\% error margin. However, the discrepancy between experimental and numerical results gets higher when inner pipe rotation plays role in the system. Nevertheless, the model can determine pressure gradient within $14 \%$ error margin for all cases.

\section{Symbols}

$\boldsymbol{A}=$ van Driest's damping constant (-)

$\boldsymbol{c}=$ Concentric radial clearence $(\mathrm{m})$

$\boldsymbol{H}=$ Half width of the slot (m)

$\boldsymbol{l}_{\boldsymbol{m}}=$ Mixing length $(\mathrm{m})$

$\boldsymbol{P}=$ Pressure $(\mathrm{Pa})$

$\boldsymbol{r}_{\boldsymbol{i}}=$ Inner pipe radius (m)

$\boldsymbol{r}_{\boldsymbol{o}}=$ Outer pipe radius (m) 


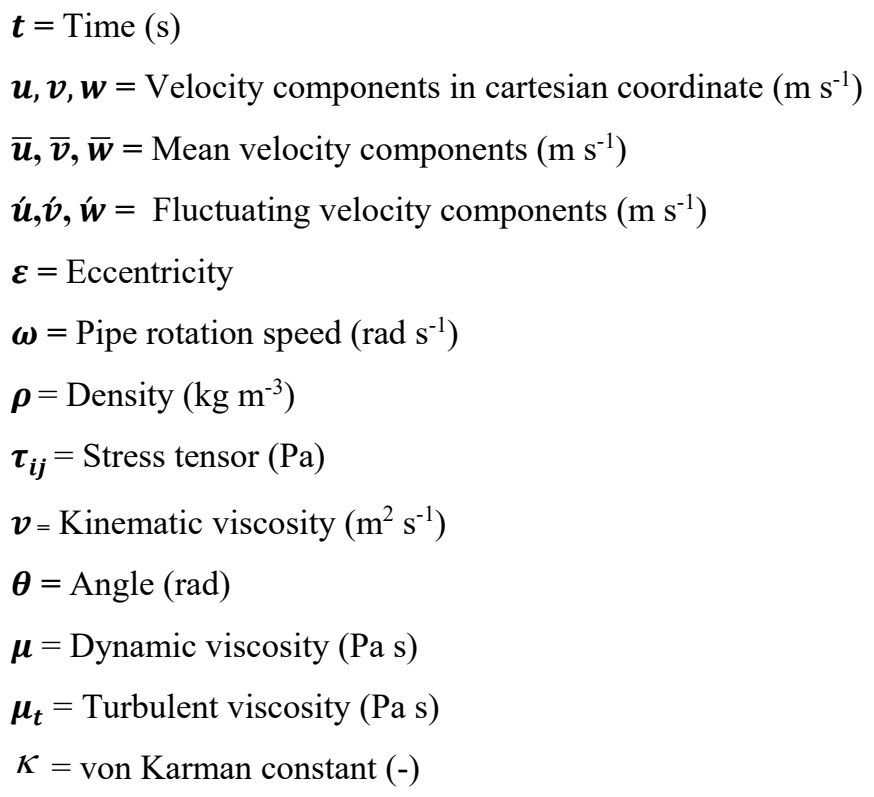

\section{References}

[1] Deissler, R. G. (1954). Analysis of turbulent heat transfer, mass transfer, and friction in smooth tubes at high Prandtl and Schmidt numbers.

[2] Deissler, R. G., \& Taylor, M. F. (1955). Analysis of fully developed turbulent heat transfer and flow in an annulus with various eccentricities. NACA Tech. Note 3451.

[3] Wolffe, R. A., \& Clump, C. W. (1963). The maximum velocity locus for axial turbulent flow in an eccentric annulus. AIChE Journal, 9(3), 424-425.

[4] Heyda, J. F. (1959). A Green's function solution for the case of laminar incompressible flow between non-concentric circular cylinders. Journal of the Franklin Institute, 267(1), 25-34.

[5] Jonsson, V. K., \& Sparrow, E. M. (1965). Results of laminar flow analysis and turbulent flow experiments for eccentric annular ducts. AIChE Journal, 11(6), 1143-1145.

[6] Jonsson, V. K., \& Sparrow, E. M. (1966). Experiments on turbulent-flow phenomena in eccentric annular ducts. Journal of Fluid Mechanics, 25(01), 65-86.

[7] Rehme, K. (1973). Simple method of predicting friction factors of turbulent flow in non-circular channels. International Journal of Heat and Mass Transfer, 16(5), 933-950.

[8] Kacker, S. C. (1973). Some aspects of fully developed turbulent flow in non-circular ducts. Journal of Fluid Mechanics, 57(03), 583-602.

[9] Usui, H., \& Tsuruta, K. (1980). Analysis of fully developed turbulent flow in an eccentric annulus. Journal of Chemical Engineering of Japan, 13(6), 445-450. 
[10] Tosun, I. (1984). Axial laminar flow in an eccentric annulus: an approximate solution. AIChE journal, 30(5), 877-878.

[11] Özgen, C., \& Tosun, I. (1987). Application of geometric inversion to the eccentric annulus system. AIChE journal, 33(11), 1903-1907.

[12] Uner, D., Ozgen, C., \& Tosun, I. (1988). An approximate solution for non-Newtonian flow in eccentric annuli. Industrial \& engineering chemistry research, 27(4), 698-701.

[13] Ogino, F., Sakano, T., \& Mizushina, T. (1987). Momentum and heat transfers from fully developed turbulent flow in an eccentric annulus to inner and outer tube walls. Heat and Mass Transfer, 21(2), 87-93.

[14] Haciislamoglu, M., \& Langlinais, J. (1990). Non-Newtonian flow in eccentric annuli. Transactions of the ASME. Journal of Energy Resources Technology, 112(3), 163-169.

[15] Nouri, J. M., Umur, H., \& Whitelaw, J. H. (1993). Flow of Newtonian and nonNewtonian fluids in concentric and eccentric annuli. Journal of Fluid Mechanics, 253, 617-641.

[16] Nouri, J. M., \& Whitelaw, J. H. (1994). Flow of Newtonian and non-Newtonian fluids in a concentric annulus with rotation of the inner cylinder. Transactions-American Society Of Mechanical Engineers Journal Of Fluids Engineering, 116, 821-821.

[17] Erge, O., Ozbayoglu, M. E., Miska, S. Z., Yu, M., Takach, N., Saasen, A., \& May, R. (2014). Effect of drillstring deflection and rotary speed on annular frictional pressure losses. Journal of Energy Resources Technology, 136(4), 042909.

[18] Mossa, M. (2006). Resistance coefficient in a smooth concentric annular pipe. Journal of Hydraulic Research, 44(6), 832-840.

[19] Erge, O., Vajargah, A. K., Ozbayoglu, M. E., \& van Oort, E. (2016, March). Improved ECD Prediction and Management in Horizontal and Extended Reach Wells with Eccentric Drillstrings. In IADC/SPE Drilling Conference and Exhibition. Society of Petroleum Engineers.

[20] Rushd, S., Shazed, A. R., Faiz, T., Kelessidis, V., Hassan, I. G., \& Rahman, A. (2017, March). CFD Simulation of Pressure Losses in Eccentric Horizontal Wells. In SPE Middle East Oil \& Gas Show and Conference. Society of Petroleum Engineers.

[21] Ulker, E., Sorgun, M., Solmus, I., \& Karadeniz, Z. H. (2017). Determination of Newtonian Fluid Flow Behavior Including Temperature Effects in Fully Eccentric Annulus. Journal of Energy Resources Technology, 139(4),04200.

[22] Bird, R. Byron, Warren E. Stewart, and Edwin N. Lightfoot (2002) Transport phenomena. John Wiley \& Sons.

[23] Erge, O., Vajargah, A. K., Ozbayoglu, M. E., \& Van Oort, E. (2015). Frictional pressure loss of drilling fluids in a fully eccentric annulus. Journal of Natural Gas Science and Engineering, 26, 1119-1129.

[24] Azouz, I., \& Shirazi, S. A. (1998). Evaluation of several turbulence models for turbulent flow in concentric and eccentric annuli. Journal of energy resources technology, 120(4), 268-275. 
[25] Iyoho, Aniekan W., and Jamal J. Azar. (1981) An accurate slot-flow model for nonNewtonian fluid flow through eccentric annuli, Society of Petroleum Engineers Journal 21.05: 565-572.

[26] Vidts, P. De and White, R. E.(1992) A semi-analytical solution method for linear partial differential equations. Computers \& Chemical Engineering, 16(10-11): 1007-1009

[27] Sheikholeslami M, Ganji D. D. (2017) Applications of Nanofluid for Heat Transfer Enhancement. Elsevier. 
